\title{
BRUCE preserves genomic stability in the male germline of mice
}

\author{
Lixiao Che ${ }^{1} \cdot$ Kris G. Alavattam ${ }^{2}$ - Peter J. Stambrook ${ }^{3}$ Satoshi H. Namekawa ${ }^{2}$ Chunying Du (i] ${ }^{1}$
}

Received: 29 June 2019 / Revised: 30 January 2020 / Accepted: 31 January 2020 / Published online: 5 March 2020

(c) The Author(s), under exclusive licence to ADMC Associazione Differenziamento e Morte Cellulare 2020

\begin{abstract}
BRUCE is a DNA damage response protein that promotes the activation of ATM and ATR for homologous recombination (HR) repair in somatic cells, making BRUCE a key protector of genomic stability. Preservation of genomic stability in the germline is essential for the maintenance of species. Here, we show that BRUCE is required for the preservation of genomic stability in the male germline of mice, specifically in spermatogonia and spermatocytes. Conditional knockout of Bruce in the male germline leads to profound defects in spermatogenesis, including impaired maintenance of spermatogonia and increased chromosomal anomalies during meiosis. Bruce-deficient pachytene spermatocytes frequently displayed persistent DNA breaks. Homologous synapsis was impaired, and nonhomologous associations and rearrangements were apparent in up to $10 \%$ of Bruce-deficient spermatocytes. Genomic instability was apparent in the form of chromosomal fragmentation, translocations, and synapsed quadrivalents and hexavalents. In addition, unsynapsed regions of rearranged autosomes were devoid of ATM and ATR signaling, suggesting an impairment in the ATM- and ATR-dependent DNA damage response of meiotic HR. Taken together, our study unveils crucial functions for BRUCE in the maintenance of spermatogonia and in the regulation of meiotic HR-functions that preserve the genomic stability of the male germline.
\end{abstract}

\section{Introduction}

BRUCE is a ubiquitously expressed protein with both ubiquitin conjugase (E2) and ligase (E3) activities [1-3]. BRUCE possesses several important pro-cell survival functions. Initially identified as a member of the inhibitor of apoptosis protein family, BRUCE promotes survival through suppressing caspase activity, thus inhibiting apoptosis [1, 4-6]. Studies of Bruce whole-body knockout (KO) mice revealed that Bruce is essential for embryogenesis [2, 5-7]. Furthermore, Bruce KO mouse embryonic

Edited by M. Oren

Supplementary information The online version of this article (https:// doi.org/10.1038/s41418-020-0513-4) contains supplementary material, which is available to authorized users.

Chunying Du

ducg@ucmail.uc.edu

1 Department of Cell and Cancer Biology, University of Cincinnati College of Medicine, Cincinnati, OH 45267, USA

2 Division of Reproductive Sciences, Cincinnati Children's Hospital Medical Center, Cincinnati, OH 45229, USA

3 Department of Molecular Genetics, University of Cincinnati College of Medicine, Cincinnati, OH 45267, USA fibroblasts evinced upregulation of the tumor suppressor p53 and displayed elevated cell death via the mitochondrial apoptosis pathway [6].

BRUCE is also a critical regulator of the DNA damage response (DDR). The DDR is a sophisticated cellular network that protects genomic stability by sensing DNA damage, activating cell cycle checkpoints, and executing DNA repair $[8,9]$. In response to DNA damage, the ATM and ATR kinases propagate signals that activate DNA repair pathways [10-13]. ATM is activated mainly in response to DNA double-strand breaks (DSBs), whereas ATR is activated chiefly in response to damaged single strand DNA (ssDNA) bound by RPA (replication protein A), a protein-DNA conformation present primarily at DNA replication forks [10-13]. In our previous reports, we demonstrated that BRUCE is a guardian of the genome. It promotes both ATM [8, 9] and ATR [14] signaling and ablation of BRUCE-induced homologous recombination (HR) defect [9]. Consequently, cells exhibit abnormal chromosomal structures (gaps, breaks, polyploidy, and telomere end-end associations) [8, 9], stalled DNA replication forks and increased firing of cryptic replication origins [14].

Preservation of genomic stability in the germline is essential for the maintenance of species. The maintenance of spermatogonia is critical for germ cell self-renewal to 
maintain the long-term production of sperm over the course of adult life. Meiosis is another critical aspect of germline maintenance. In meiosis, developmentally programmed DNA DSBs occur to initiate meiotic recombination [15], which promotes the reshuffling of genetic material between maternal and paternal alleles for producing genetic variation in the next generation [16, 17]. Meiotic DNA DSBs are potential sources of genomic instability and, thus, necessitate the existence of specialized mechanisms to preserve germline genomic stability. However, in spermatogonia and in meiotic spermatocytes, the mechanisms by which genomic stability is preserved remain a mystery.

To gain more mechanisms that preserve genomic stability in the germline, we have investigated the function of BRUCE and identified novel functions for BRUCE in spermatogonial maintenance and meiotic DDR. Our work demonstrates that BRUCE is a critical regulator of genomic stability in the germline.

\section{Materials and methods}

\section{Mouse strains and husbandry}

Mice harboring two conditional Bruce alleles (Bruce loxp/loxp $^{\text {lo }}$ were crossed with UBC-Cre-ER ${ }^{\mathrm{T} 2}$-positive mice (JAX, 008085). All mice were backcrossed to pure C57B1/6 mice. Breeding was in keeping with IACUC guidelines at the University of Cincinnati.

\section{Induction of Bruce depletion in mice}

To recombine the BRUCE floxed allele using the Cre$\mathrm{ER}^{\mathrm{T} 2}$ transgenic system, tamoxifen (MP Biomedicals) was solubilized in corn oil (Sigma, C8267) to a concentration of $20 \mathrm{mg} / \mathrm{mL}$. Starting at P7, $10 \mu \mathrm{L}$ tamoxifen solution was delivered into each mouse pup via intraperitoneal injection (i.p.) for three consecutive days (P7-P9). Tamoxifen administration was performed before genotyping to ensure blinded. Mice were then sacrificed and assayed randomly at the ages of postnatal day 20 (P20) and 7 weeks (P49).

\section{PCR analyses}

DNA was extracted from the indicated mouse tissues and subjected to dual PCR for the Bruce floxed allele and the cKO allele. The following primers were used: SC1 (ATG TGCTGGGGTGGCTCATCAAC) and NDEL2 (GCCCTG GGCTATTACTACACATAAGCC). Locations of primer binding are shown in the schematic of the Bruce floxed and cKO regions (Fig. 1a).

\section{Western blot analyses}

Tissues from Bruce Ctrl and cKO mice were homogenized and lysed in RIPA buffer (20 mM Tris-HCI pH 8.0, 150 $\mathrm{mM} \mathrm{NaCl}, 2 \mathrm{mM}$ EDTA, $1 \% \mathrm{NP} 40,1 \%$ sodium deoxycholate, $0.1 \%$ SDS) supplemented with protease inhibitors (Thermo Scientific, 88668). After clearing via centrifugation, the supernatant was collected for western blot analyses.

\section{Histology, TUNEL staining, and immunohistochemistry}

Testes and epididymides were fixed overnight at room temperature in $10 \%$ neutral-buffered formalin, embedded in wax, sectioned at 5-7 $\mu \mathrm{m}$ thickness, and stained with hematoxylin and eosin (H\&E). Terminal deoxynucleotidyl transferase dUTP nick end labeling (TUNEL) staining was performed following the manufacturer's instructions (Millipore, S7110). For immunohistochemistry and immunofluorescence analyses, testis slides were antigen-retrieved with sodium citrate buffer ( $\mathrm{pH}$ 6.0) and incubated with primary antibodies overnight at $4{ }^{\circ} \mathrm{C}$. Then, slides were incubated with biotin-conjugated secondary antibodies, which were detected by 3,3'-Diaminobenzidine staining using an immunohistochemistry VECTASTAIN ABC kit (Vector Laboratories, PK-6100) or via Alexa-conjugated secondary antibodies (Thermo Fisher). For immunofluorescence analyses, slides were counterstained with 4',6diamidino-2-phenylindole (DAPI).

\section{Immunofluorescence microscopy analyses of spermatocyte chromosome spreads}

Surface-spread nuclei were prepared as described previously [18]. Briefly, seminiferous tubules were incubated in hypotonic extraction buffer $(30 \mathrm{mM}$ Tris, $50 \mathrm{mM}$ sucrose, $17 \mathrm{mM}$ trisodium citrate dihydrate, $5 \mathrm{mM}$ EDTA, $0.5 \mathrm{mM}$ DTT, $1 \times$ protease inhibitor cocktail; adjusted to $\mathrm{pH} 8.2$ with $\mathrm{HCl}$ and/or $\mathrm{NaOH}$ ) on ice for $\sim 2-3 \mathrm{~h}$, gently minced in $100 \mathrm{mM}$ sucrose, and spread on slides dipped in fixation solution (2\% PFA, $0.15 \%$ Triton X-100, 0.02\% SDS; adjusted to $\mathrm{pH} 9.2$ with sodium borate buffer). Slides were incubated in a humid chamber for $2 \mathrm{~h}$, dried $\sim 30 \mathrm{~min}$, and then washed in $0.4 \%$ Photo-Flo 200 (Kodak).

Immunofluorescence staining was performed following blocking in $10 \%$ BSA; primary antibodies were incubated overnight at $4{ }^{\circ} \mathrm{C}$, and Alexa-conjugated secondary antibodies incubated at room temperature for $\sim 1 \mathrm{~h}$ in darkness. Slides were mounted with coverslips using mounting medium containing DAPI. Images were acquired with a fluorescence microscope (Zeiss or Nikon) and processed with ImageJ (NIH) and/or NIS-Elements Basic Research 
A

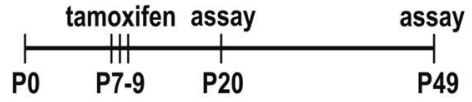

B Testis

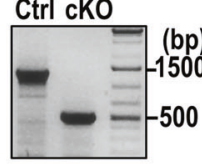

C
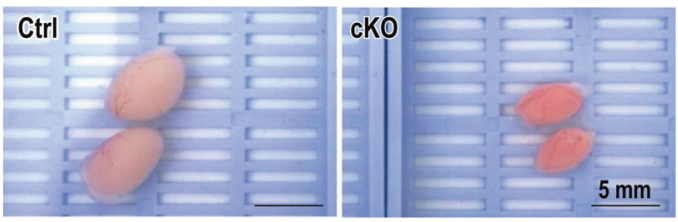

D

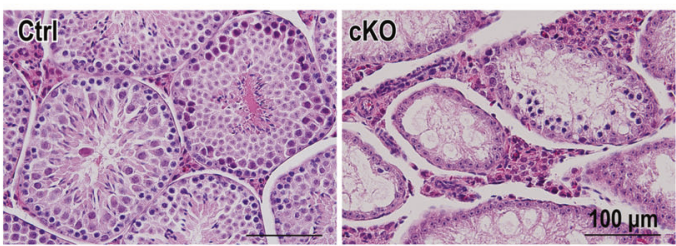

E

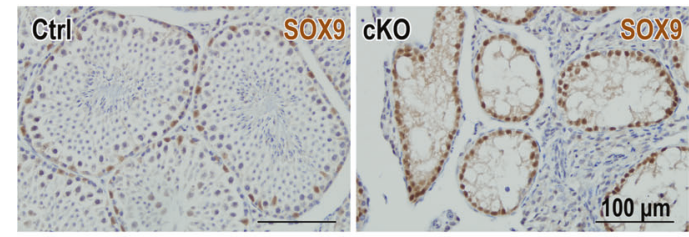

$\mathbf{F}$
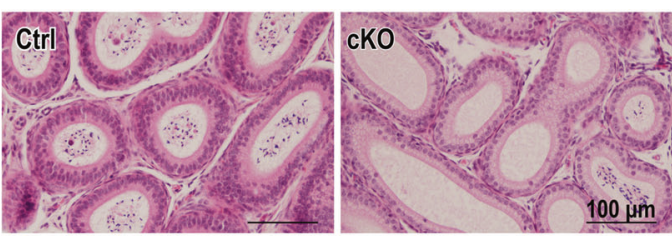
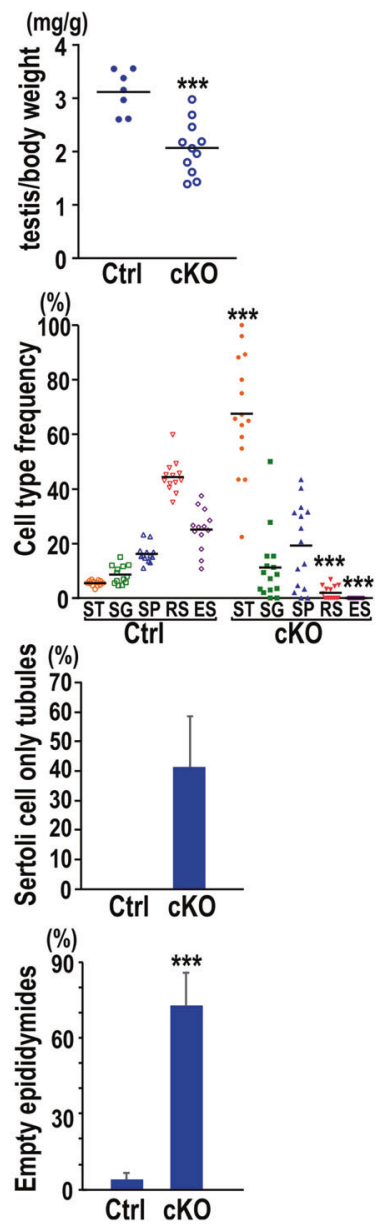

Fig. 1 Conditional inactivation of Bruce in mice results in reduced testis size and germ cell depletion. a Tamoxifen treatment scheme to

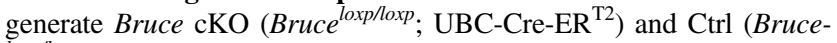
loxp/loxp $)$ mice. Mouse pups were exposed to tamoxifen via intraperitoneal injections once a day on postnatal days 7, 8, and 9 (P7-9). For analyses, all mice were sacrificed at 7 weeks of age (P49). b Evaluation of KO efficiency in testes via PCR (left) and western blot (right) analyses. The PCR primers SC1 and NDEL2 were used (see Fig. S1A, "Materials and methods"). c Photos of testes at 7 weeks of age show smaller testis sizes in Bruce cKO mice compared with Ctrl mice. Testis/body weight ratios are shown in a dot plot to the right. Data are aggregated from $7 \mathrm{Ctrl}$ and $11 \mathrm{cKO}$ mice. Horizontal line, mean. Student's $t$ test, $* * * p<0.001$. d Hematoxylin and eosin (H\&E) analyses of testis sections at 7 weeks of age. Frequencies of different cell types in Bruce Ctrl and cKO testes are shown in a dot plot to the right. Data are aggregated from two independent Bruce Ctrl-cKO littermate pairs. Horizontal line, mean. Student's $t$ test, $* * * p<0.001$. ST Sertoli cells; SG spermatogonia; SP spermatocytes; RS round spermatids; ES elongating spermatids. e Immunohistochemistry (IHC) of SOX9, a marker of Sertoli cells, in Bruce Ctrl and cKO testis sections at 7 weeks of age. Frequencies of $\mathrm{Ctrl}$ and cKO tubules bearing only Sertoli cells are shown in a bar graph to the right. Data are aggregated from two independent Bruce Ctrl-cKO littermate pairs (tubule number, $\mathrm{Ctrl}=41, \mathrm{cKO}=76$ ). Error bar, standard deviation. Student's $t$ test, $*_{* *} p<0.001$. f H\&E analyses of epididymis sections from Bruce Ctrl and cKO mice at 7 weeks of age. Frequencies of Ctrl and cKO empty epididymides are shown in a bar graph to the right. Data are aggregated from three independent Bruce Ctrl-cKO littermate pairs (epididymal ducts number, $\mathrm{Ctrl}=47, \mathrm{cKO}=214$ ). Error bar, standard deviation. Student's $t$ test, $* * * p<0.001$. 
(Nikon), CorelDRAW X7, Photoshop (Adobe), and Illustrator (Adobe).

Fluorescence intensity of ATR signals in spermatocyte chromosome spreads were measured with NIS-Elements Basic Research software (Nikon) following a published workflow [19] for quantitative imaging of surface-spread spermatocyte nuclei. Minor alterations to the workflow are described here. Briefly, regions of interest (ROIs) were drawn around pachytene spermatocyte XY body regions, denoted as "XY" in Fig. S4D, and pachytene spermatocyte nuclear regions excluding XY bodies, denoted as "Au." (for "autosome regions") in Fig. S4D. Since XY bodies are not present in zygotene spermatocytes, Au. ROIs were drawn around the whole perimeters of zygotene nuclei. To facilitate comparisons between Bruce Ctrl and cKO samples, we normalized fluorescence signals on a relative scale (0-2.0). To do so, we calculated the mean of all Bruce Ctrl mid pachytene $\mathrm{XY}$ body ROI signals, ' $\left(\Sigma{ }^{\mathrm{Ctrl}} \mathrm{XY} \mathrm{MP}_{\mathrm{MP}}\right) \div(\mathrm{N}$ $\left.{ }^{\mathrm{C} t r l} \mathrm{XY} \mathrm{MP}_{\mathrm{MP}}\right)=\mu{ }^{\mathrm{Ctrl}} \mathrm{XY} \mathrm{YP}_{\mathrm{MP}}$; then, we divided each individual Ctrl and cKO XY and Au. ROI measurement by ' $\mu$ ${ }^{\mathrm{Ctrl}} \mathrm{XY}_{\mathrm{MP}}$ ' as in, for example, the following calculation for an individual Au. ROI measurement of a Bruce cKO late pachytene spermatocyte: '(ROI $\left.{ }^{{ }^{\mathrm{KO}} \mathrm{Au} \cdot \mathrm{LP}}\right) \div\left(\mu^{{ }^{\mathrm{Ctrl}} \mathrm{XY}} \mathrm{MP}_{\mathrm{MP}}\right)$ '. For each sample, this provided a value for ROI signals termed the "relative mean fluorescence intensity" (RMFI),

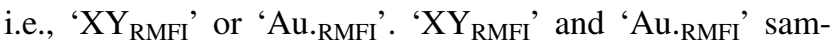
ples were collected into separate pools by genotype. The pools were then subdivided by the following stages of meiotic prophase I: the zygotene stage ( $\mathrm{Z})$, the early pachytene stage (EP), the mid pachytene stage (MP), and the late pachytene stage (LP). Using Excel (Microsoft) and Prism 6.0 or 7.0 (GraphPad), statistical analyses were run on the subdivided pools, and mean + standard error for each subdivided pool was derived and plotted.

\section{RNA FISH}

Slides that conserve the gross three-dimensional nuclear organization, chromatin organization, and morphology of mouse male germ cells were prepared as described [20, 21]. Using these slides, Cot-1 RNA FISH was performed as described [20, 22], and gene-specific RNA FISH for Lamp2 probe conjugated to the fluorophore $\mathrm{Cy} 3$ was performed as described [22, 23].

\section{Data analysis}

Images of Bruce $\mathrm{Ctrl}$ and cKO chromosomes were randomized and subject to blinded measurements. Details for statistical analyses and sample sizes are described in relevant portions of the figures, figure legends, supplementary figures, and/or supplementary figure legends. In predetermining sample sizes, we sought to analyze a minimum of two independent Bruce Ctrl-cKO littermate pairs; many analyses drew on three or more Bruce Ctrl-cKO littermate pairs. No statistical calculations were used to predetermine sample sizes, and no data were excluded from analyses. As noted, results were expressed as either means, means + standard deviation, or means + standard error. Unless otherwise noted, the statistical significance of observed differences was derived from two-tailed Student's $t$ tests; for Fig. S4C, the statistical significance of observed differences was derived from one-way ANOVA and Tukey's method posttest. Measurements were recorded in Excel (Microsoft) and Prism 6.0 or 7.0. Statistical tests were performed with Excel and Prism 6.0 or 7.0 .

\section{Antibodies}

For western blots and immunohistochemistry: BRUCE (Bethyl, A300-367A, 1:1000); Tubulin (Sigma, T9026, 1:4000); SOX9 (Cell Signaling, 82630, 1:500); Cleaved Caspase-3 (Cell Signaling, 9664, 1:200).

Primary antibodies for immunofluorescence: c-KIT (Cell Signaling, 3074, 1:400); PLZF (SCBT, sc-28319, 1:200); SYCP3 (Novus, NB300-232, 1:200; SCBT, sc-74569, 1:400); SYCP1 (Novus, NB300-229, 1:200); $\gamma \mathrm{H} 2 \mathrm{AX}$ (Millipore, 05-636, 1:10000); RNA Pol II (Millipore, 05-952, 1:200); pATM (Calbiochem, DR1002, 1:200); ATR (SCBT, sc-515173, 1:100); BRCA1 (generated in the Namekawa lab [24], 1:2000); RAD51 (Calbiochem, PC130, 1:200); RPA1 (gift from the Ingles lab, 1:100); HORMAD1 (gift from the Tóth lab, 1:500); and HORMAD2 (gift from the Tóth lab, 1:800).

Secondary antibodies for immunofluorescence: Alexa Fluor 488 (Thermo Fisher); Alexa Fluor 555 (Thermo Fisher); Alexa Fluor 594 (Thermo Fisher).

\section{Results}

\section{Generation of Bruce conditional knockout (cKO) mice}

To investigate the function of BRUCE in the germline, we generated a Bruce cKO mouse model using a floxed mouse strain in which Bruce exon 2 is flanked with loxp sites [14] (Fig. S1A). The floxed Bruce strain was bred with a strain of transgenic mice in which Cre-ER ${ }^{\mathrm{T} 2}$ is driven by the ubiquitous UBC (ubiquitin C) promoter (UBC-Cre-ER ${ }^{\mathrm{T} 2}$; Fig. S1B) [25]. To induce the cKO of Bruce, mouse pups were treated with tamoxifen on postnatal days 7-9 (P7-P9) and sacrificed on P20 or 7 weeks of age (P49) for phenotypic examinations (Fig. 1a). The cKO of Bruce was highly efficient throughout the body (Fig. S1C, D), including in the testes (Fig. 1b). Importantly, this transgenic breeding 
scheme bypasses the embryonic lethality of Bruce KO, thereby establishing a new Bruce cKO mouse model that enables investigations into the functions of BRUCE in adult mice.

\section{Spermatogenic failure in Bruce cKO testes}

Following tamoxifen induction on P7-P9, neonate Bruce cKO mice develop normally based on appearance and body size similar to that of Bruce Ctrl littermates (data not shown). At 7 weeks of age, no discernible changes were found except for the testes: cKO testes were much smaller than those of Ctrl with a significant reduction in the testis/ body weight ratio (Fig. 1c). Histological examinations found that $\mathrm{cKO}$ seminiferous tubules were significantly reduced in size, and only 1-3 layers of cells remained (Fig. 1d). By quantification of the frequency of each germ cell type, we noted slightly increased proportions of spermatocytes and spermatogonia, and dramatically decreased round and elongating spermatids (Fig. 1d). This relative increase in spermatogonia and spermatocytes could be due to the massive depletion of postmeiotic germ cells. In addition, more than $60 \%$ of cells remaining in Bruce cKO seminiferous tubules were Sertoli cells (Fig. 1d), confirmed by immunostaining for SOX9, a Sertoli cell marker (Fig. 1e) [26, 27]. The proportion of the tubules having only Sertoli cells was $41.4 \%$ (Fig. 1e). Consequently, the cKO epididymides were largely empty (Fig. 1f).

Through IHC analyses of Bruce Ctrl mice, we determined that BRUCE protein was abundant in spermatogonia, spermatocytes, and spermatids (Fig. 2a). Thus, we sought to determine the function of BRUCE in differentiated and undifferentiated spermatogonia by PLZF $[28,29]$ and c-KIT antibodies [30, 31], respectively. These analyses showed a paucity of undifferentiated (Fig. 2b) and differentiating (Fig. 2c) spermatogonia in cKO, supporting a critical function of BRUCE not only for spermatocyte development but also for spermatogonial maintenance.

The severe germ cell loss is intriguing since Bruce is an inhibitor of apoptosis, and the elimination of Bruce may activate the apoptotic program in the testes. To assess this possibility, we examined the stage of spermatogenesis in which severe germ cell loss occurs by TUNEL and Cleaved Caspase-3. In comparison to Ctrl, we observed a dramatic increase in TUNEL (Fig. 2d) and Cleaved Caspase-3 signals (Fig. 2e) in cKO. Based on cell morphology, the majority of apoptotic cells appeared to be spermatocytes (Fig. 2d, e). Because the selective deletion of Bruce in early embryonic stages presents a highly technical challenge, we were unable to determine the function of Bruce in primordial germ cells or oocytes in meiotic prophase I.

To expand our understanding of BRUCE's roles in juvenile testis development, we examined P20 mice. In contrast to the Bruce Ctrl testes, far fewer spermatocytes were present in the cKO (Fig. S2A), and such a scarcity was associated with increased apoptosis as indicated by TUNEL (Fig. S2B). IHC analyses showed that BRUCE expression was ablated in cKO testes (Fig. S2C).

\section{Meiotic DNA repair deficiencies in Bruce cKO spermatocytes}

Next, to identify the cause of germ cell loss in the absence of Bruce, we examined the spermatogenesis at 7 weeks of age. During meiotic prophase I, spermatocytes progress sequentially through the following stages (Fig. 3a): the leptotene stage, in which chromosomes begin to condense and axial elements of the synaptonemal complex (SC), a proteinaceous polymer that functions as a scaffold between chromosomes [16], first appear; the zygotene stage, in which homologous chromosomes begin to pair and synapse; the pachytene stage, in which homologous pairing and synapsis have completed; and the diplotene stage, in which homologous chromosomes segregate from each other. Meiotic DSBs are generated during the leptotene stage, at which time meiotic recombination begins. The repair of DSBs is completed in the zygotene and pachytene stages. Meiosis requires the full synapsis of homologs via the SC. To evaluate meiotic progression, spermatocytes were immunostained against SYCP3, an axial element of the SC; the judgment of SYCP3 patterns allows precise, accurate determination of stages of meiotic prophase I [18]. We also immunostained against histone H2AX phosphorylated at serine $139(\gamma \mathrm{H} 2 \mathrm{AX})$, a marker for DNA damage signaling that is used to characterize meiotic prophase I $[18,32]$. We observed patches of $\gamma \mathrm{H} 2 \mathrm{AX}$ immunofluorescence in leptotene and zygotene spermatocytes in both Ctrl (Fig. 3b, c) and cKO (Fig. 3d, e), suggesting the successful generation of meiotic DSBs in Bruce cKO cells.

We previously showed that BRUCE promotes ATM signaling in response to DSBs induced by ionizing radiation [8, 9]. In meiosis, active (phosphorylated) ATM (pATM), which senses DNA damage, blocks the function of SPO11, the topoisomerase II-like enzyme that catalyzes programmed DNA DSBs in leptotene spermatocytes [33]. Thus, to assess the status of ATM signaling in response to programmed meiotic DNA DSBs in the cKO, we immunostained chromosome spreads for pATM and found no differences between $\mathrm{Ctrl}$ and cKO leptotene spermatocytes (Fig. S3), suggesting that ATM signaling appears normal in Bruce cKO spermatocytes in the leptotene stage. Interestingly, in comparison to Ctrl (Fig. 3f, h), $\gamma \mathrm{H} 2 \mathrm{AX}$ signals were retained at portions of autosomes in Bruce cKO pachytene and diplotene spermatocytes (Fig. 3g, i), which was three folds more in cKO spermatocytes than Ctrl 

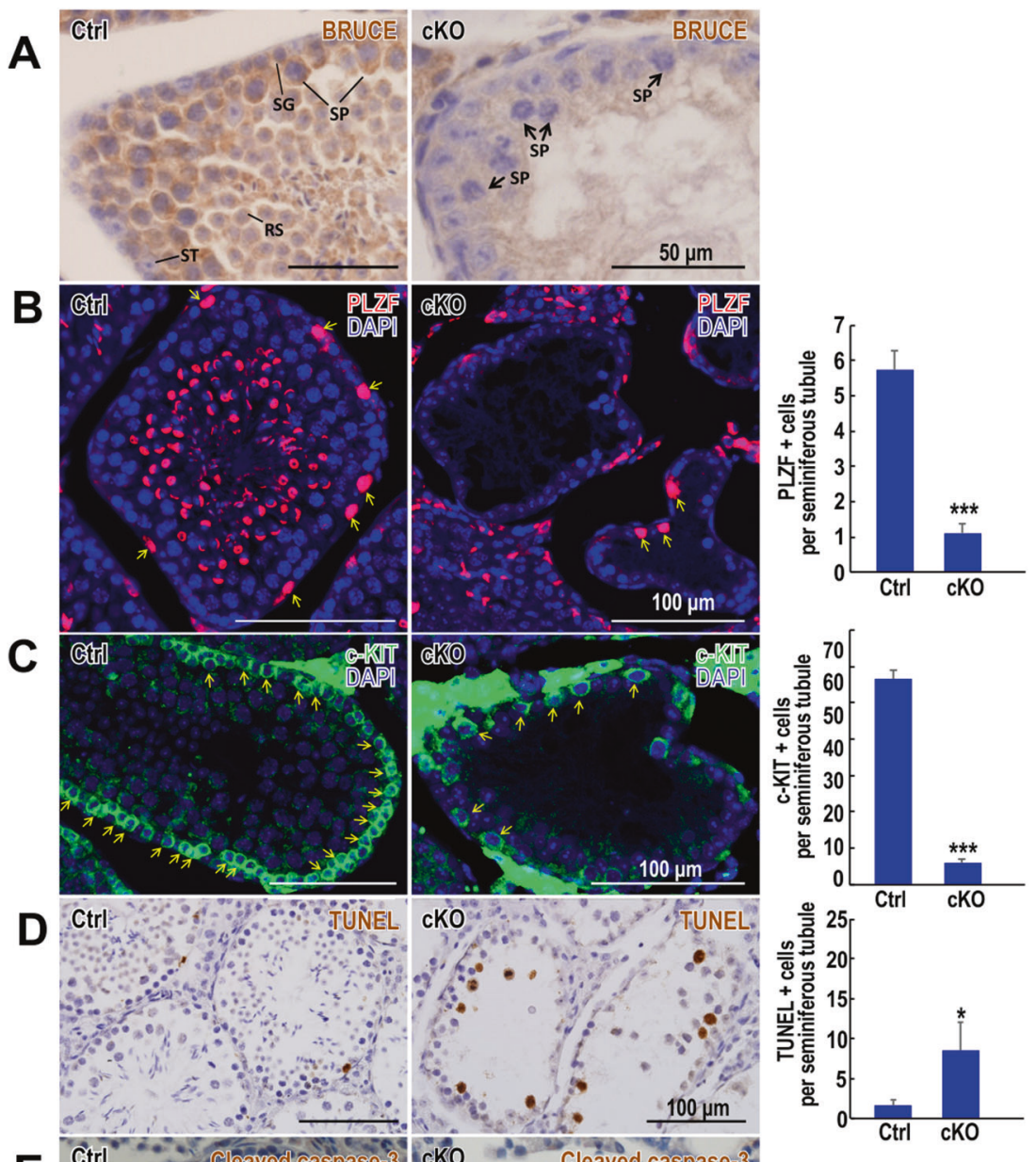

E
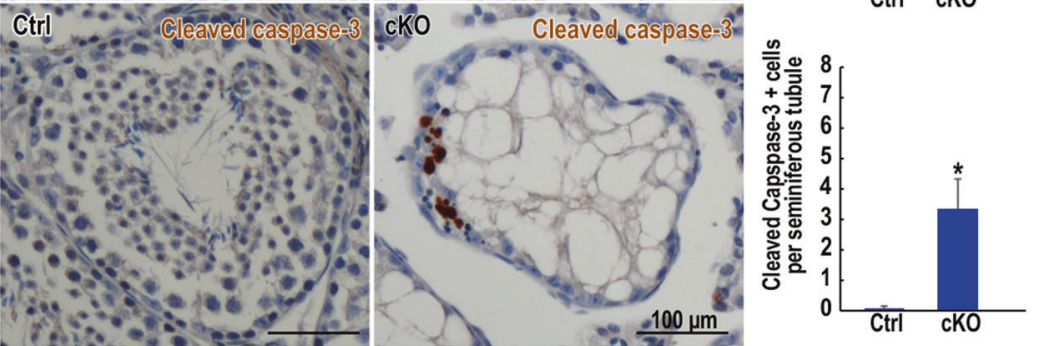

Fig. 2 Conditional inactivation of Bruce in mice results in apoptosis of germ cells. a Immunohistochemistry (IHC) of Bruce confirms the high efficiency of Bruce KO. Arrows indicate spermatocytes devoid of Bruce protein expression in a cKO testis at 7 weeks of age. SG: spermatogonium; ST Sertoli cells; SP spermatocytes; RS round spermatids. Representative images from two independent Bruce CtrlcKO littermate pairs. b Immunofluorescence (IF) staining of Bruce $\mathrm{Ctrl}$ and cKO testis sections at 7 weeks of age with PLZF, a marker of undifferentiated spermatogonia. Arrows indicate PLZF-positive spermatogonia. Quantification of PLZF-positive cells is shown in a bar graph to the right. Data are aggregated from two independent Bruce Ctrl-cKO littermate pairs (tubules number, $\mathrm{Ctrl}=35, \mathrm{cKO}=55$ ). Error bar, standard error. Student's $t$ test, $* p<0.05$. c IF staining of Bruce Ctrl and cKO testis sections at 7 weeks of age with c-KIT, a marker of differentiating spermatogonia. Arrows indicate c-KITpositive $(+)$ spermatogonia. Number of c-KIT + cells per tubule is shown in a bar graph to the right. Data are aggregated from two independent Bruce Ctrl-cKO littermate pairs (tubules number, $\mathrm{Ctrl}=$ $42, \mathrm{cKO}=45)$. Error bar, standard error. Student's $t$ test, $* * * p<$ 0.001. d Terminal deoxynucleotidyl transferase dUTP nick end labeling (TUNEL) staining of Bruce Ctrl and cKO testis sections at 7 weeks of age. Staining indicated increased numbers of TUNELpositive cells in Bruce cKO. Quantification of TUNEL-positive (+) cells is shown in a bar graph to the right. Data are aggregated from three independent Bruce Ctrl-cKO littermate pairs (tubules number, $\mathrm{Ctrl}=100, \mathrm{cKO}=55$ ). Error bar, standard error. Student's $t$ test, ${ }^{*} p<$ 0.05. e IHC of Bruce Ctrl and cKO testis sections at 7 weeks of age with Cleaved Caspase-3, a marker of apoptotic cells. Quantification of Cleaved Caspase-3-positive $(+)$ cells is shown in a bar graph to the right. Data are aggregated from two independent Bruce Ctrl-cKO littermate pairs (tubules number, $\mathrm{Ctrl}=13, \mathrm{cKO}=8$ ). Error bar, standard error. Student's $t$ test, ${ }^{*} p<0.05$. 

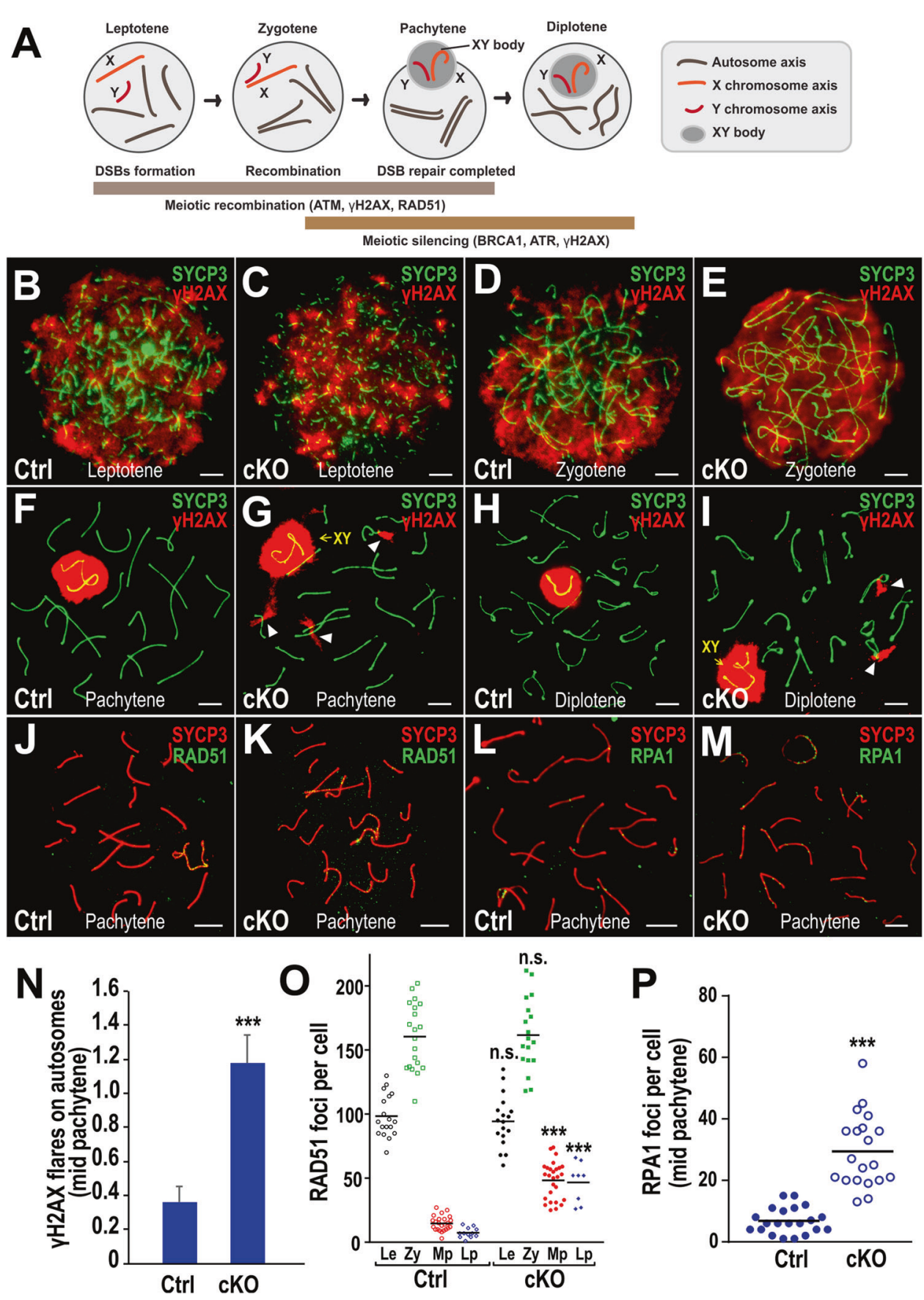

(Fig. 3n), indicating an aberrant persistence of unrepaired DNA DSBs and suggesting an HR defect.

To verify this HR defect, we examined RAD51, an HR marker for recombination intermediates [34], and RPA1, an ssDNA stabilizer to coordinate recombination [35]. Comparing to Ctrl, we observed the marked retention of RAD51 foci associated with pachytene chromosome axes in the cKO (Fig. 3j, k, o), a strong indication of persistent DSBs. Similar results were found with RPA1 foci (Fig. 31, m, p). Together, these data further implicate an HR defect in Bruce cKO spermatocytes.

\section{Chromosome breakage/fragmentation and lateral axial defects in Bruce cKO spermatocytes}

To evaluate the extent of defective HR in Bruce cKO spermatocytes, we assessed crossover, the outcome of HR by immunostaining chromosome spreads for MLH1, a mismatch repair factor and meiotic recombination marker that localizes to the sites of crossovers [36]. We observed no distinguishable difference in the numbers of MLH1 foci in Bruce Ctrl versus cKO spermatocytes (Fig. $4 \mathrm{a}-\mathrm{c}$ ), indicating that Bruce is not critical for crossover formation. 
4 Fig. 3 Bruce cKO spermatocytes show increase in unrepaired DSBs and defective synapsis in meiotic prophase I. a Diagram illustrating the major molecular events during meiotic prophase I. Immunofluorescence (IF) staining of the synaptonemal complex component SYCP3 (green) and the DNA double-strand break marker $\gamma \mathrm{H} 2 \mathrm{AX}$ (red) in chromosome spreads of Bruce Ctrl (b) and cKO (c) leptotene spermatocytes. Representative images from four independent Bruce Ctrl-cKO littermate pairs. Bars, $5 \mu \mathrm{m}$. IF staining of the SYCP3 (green) and $\gamma \mathrm{H} 2 \mathrm{AX}$ (red) in chromosome spreads of Bruce $\mathrm{Ctrl}(\mathbf{d})$ and $\mathrm{cKO}$ (e) spermatocytes at zygotene. Representative images from four independent Bruce Ctrl-cKO littermate pairs. Bars, $5 \mu \mathrm{m}$. IF staining of the SYCP3 (green) and $\gamma \mathrm{H} 2 \mathrm{AX}$ (red) in chromosome spreads of Bruce $\mathrm{Ctrl}(\mathbf{f})$ and $\mathrm{cKO}(\mathbf{g})$ pachytene spermatocytes. Excessive $\gamma \mathrm{H} 2 \mathrm{AX}$ signals (arrowheads in $\mathbf{g}$ ) were persistent on cKO autosomes, implying unrepaired DSBs. Representative images from four independent Bruce Ctrl-cKO littermate pairs. Bars, $5 \mu \mathrm{m}$. IF staining of the SYCP3 (green) and $\gamma \mathrm{H} 2 \mathrm{AX}$ (red) in chromosome spreads of Bruce $\mathrm{Ctrl}$ (h) and cKO (i) diplotene spermatocytes. Note that $\gamma \mathrm{H} 2 \mathrm{AX}$ signals were persistent on cKO autosomes (arrowheads in I). Representative images from four independent Bruce Ctrl-cKO littermate pairs. Bars, $5 \mu \mathrm{m}$. (j, k) IF staining of SYCP3 (red) and recombination marker RAD51 (green) in Bruce Ctrl (j) and cKO (k) mid pachytene spermatocytes. Representative images from three independent Bruce Ctrl-cKO littermate pairs. Bars, $5 \mu \mathrm{m}$. IF staining of SYCP3 (red) and recombination marker RPA1 (green) in Bruce Ctrl (l) and cKO (m) mid pachytene spermatocytes. Representative images from two independent Bruce Ctrl-cKO littermate pairs. Bars, $5 \mu \mathrm{m}$. $\mathbf{n}$ Comparison of the numbers of $\gamma \mathrm{H} 2 \mathrm{AX}$ flares in Bruce Ctrl and cKO mid pachytene spermatocytes. Data are aggregated from three independent Bruce Ctrl-cKO littermate pairs (spermatocytes number, $\mathrm{Ctrl}=80, \mathrm{cKO}=111$ ). Error bar, standard error. Student's $t$ test, $* * * p<0.001$. o Quantification of RAD51 foci in Bruce Ctrl and cKO spermatocytes during prophase I. Le leptotene; Zy zygotene; Mp mid pachytene; Lp late pachytene. Data are aggregated from three independent Bruce Ctrl-cKO littermate pairs. Horizontal line, mean. Student's $t$ test, n.s. not significant, $p>$ 0.05; *** $p<0.001$. p Quantification of RPA1 foci in Bruce Ctrl and cKO mid pachytene spermatocytes. Bruce cKO mid pachytene spermatocytes evinced persistent RPA1 foci. Data are aggregated from two independent Bruce Ctrl-cKO littermate pairs. Horizontal line, mean. Student's $t$ test, $* * * p<0.001$.

Yet despite normal crossover formation, it is possible that the HR defect in Bruce cKO manifests in other ways. Aberrant accumulation of DNA breaks coupled with asynapsis is detrimental to genomic stability in germ cells. Indeed, we identified severe chromosome breakage/fragmentation and lateral axial damage in Bruce cKO pachytene spermatocytes. The chromosomal breakage/fragmentation was characterized by the presence of one or more extra pieces of fragmented chromosome in the spermatocyte nuclei (Fig. 4d, dashed outline), in addition to the exact 20 chromosome pairs (19 autosome pairs plus the sex chromosomes). Fragmented chromosomes were surrounded by intense $\gamma \mathrm{H} 2 \mathrm{AX}$ fluorescence, indicative of DNA damage. In addition, portions of lateral axial elements of the SC exhibited relatively weak SYCP3 immunofluorescence signals, suggesting damaged lateral axial structures (Fig. 4e, f). In cKO, $4.1 \%$ of the observed pachytene spermatocytes exhibited chromosomal breakage/fragmentation and axial damage, a significantly higher proportion versus Ctrl
(Fig. 4g). Together, these data demonstrate a function for Bruce as a guardian of genome integrity in spermatocytes.

\section{Impaired ATM and ATR signaling with chromosomal radials in Bruce cKO spermatocytes}

The chromosomal breakage/fragmentation and SC lateral axial defects described above are consistent with chromosomal fragility. In human male germ cells, chromosomal fragility can produce chromosomal rearrangements, including translocations [37, 38]. In addition, formation of quadrivalents is a hallmark of translocation [39]. Indeed, Bruce deficiency induces translocations between nonhomologous chromosomes (Fig. 5a-c). These include three distinct forms of translocation: reciprocal (Fig. 5a), nonreciprocal (Fig. 5b), and open conformation that closely resembles a Robertsonian translocation (Fig. 5c), the most common form of translocation in humans [37, 38]. Chromosomal translocations can be generated by error-prone repair of the DNA breaks via the ligation of broken nonhomologous chromosomes [40, 41]. The quadrivalents exhibited persistent DNA breaks and incomplete meiotic recombination as evinced by intense $\gamma \mathrm{H} 2 \mathrm{AX}$ signals in their surrounding chromatin (Fig. 5a-c), and by intense RAD51 foci on the chromosome axes (Fig. 5d). However, pATM, a sensor of DNA damage, was absent at radial structures (Fig. 5e), indicating an impaired ATM function at translocated chromosomes and suggesting the promotion of chromosomal translocations via accumulated DNA DSBs. We have provided a detailed comparison of the chromosomal defects in cKO versus Ctrl (Fig. 5f).

ATR signaling is crucial for synapsis and HR in prophase I $[42,43]$. In pachytene spermatocytes, efficient ATR activity on unsynapsed chromosome regions is believed to form the basis of meiotic silencing of unsynapsed chromatin (MSUC) [44-46]. Normally MSUC is confined to unsynapsed sex chromosomes to facilitate meiotic sex chromosome inactivation (MSCI) [44]. This process is regulated by DDR pathways centered on ATR, $\gamma \mathrm{H} 2 \mathrm{AX}$, and MDC1 [24, 45, 46]. In Ctrl, ATR forms discrete foci on the unsynapsed axes of chromosomes during zygotene (Fig. 6a) and remains exclusively on the sex chromosomes at pachytene (Fig. 6b). Although ATR signals in Bruce cKO zygotene spermatocytes were comparable to those in Ctrl (Fig. 6c), ATR were absent from the unsynapsed segments of quadrivalents in Bruce cKO mid and late pachytene spermatocytes (Fig. 6d, e), and reduced on the sex chromosomes (Fig. S4A-C). The tumor suppressor BRCA1 participates in the same MSUC process [47, 48]. In Ctrl, BRCA1 accumulated on the unsynapsed axes of autosomes in zygotene (Fig. 6f), and was restricted to the unsynapsed axes of the sex chromosomes in pachytene (Fig. 6g). In $\mathrm{cKO}$, the zygotene BRCA1 patterns were the same as the 

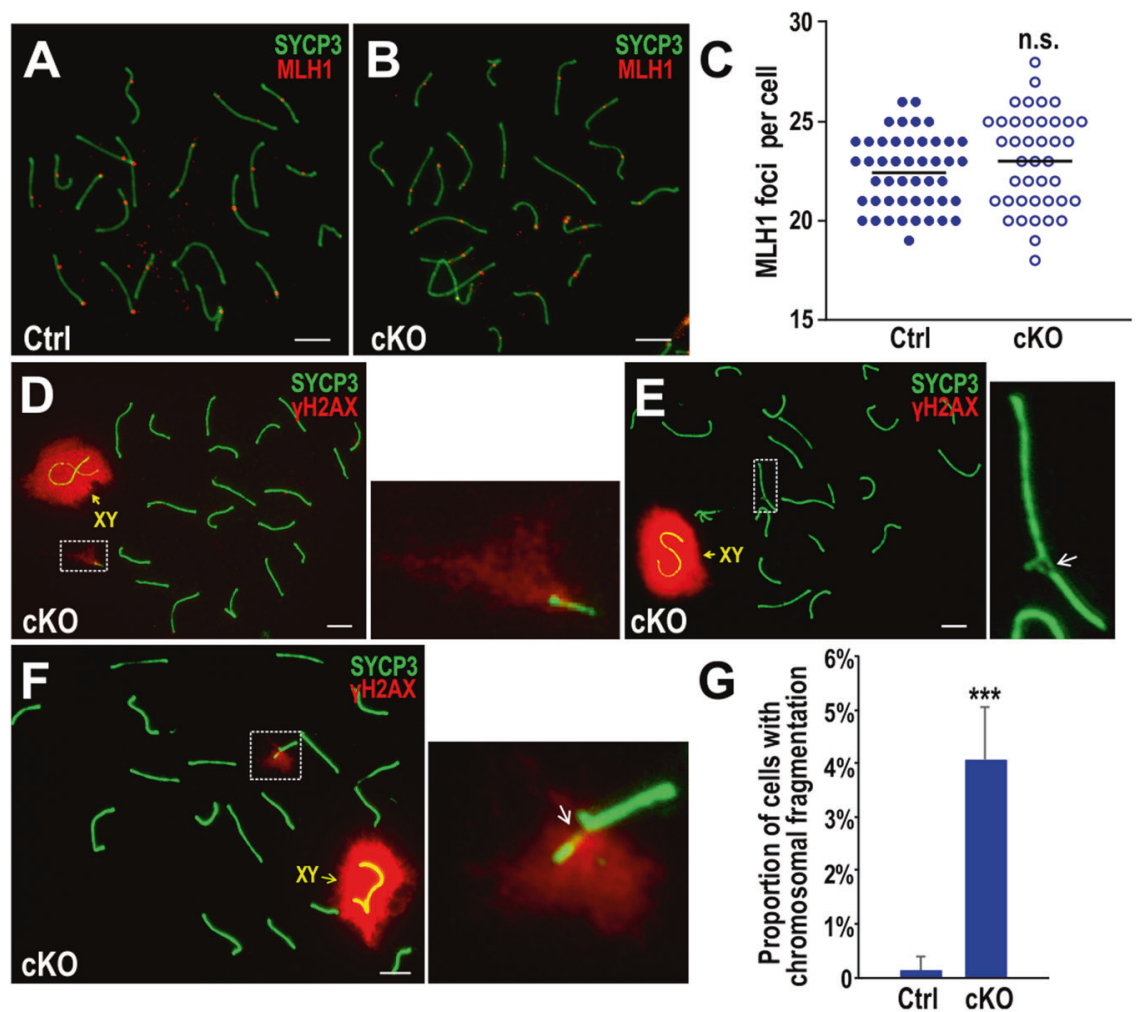

Fig. 4 Bruce cKO spermatocytes show chromosomal fragmentation and lateral axial damage. a, b Chromosomal spread of Bruce Ctrl and cKO spermatocytes immunostained with SYCP3 (green) and MLH1 (red) antibodies. Representative images from three independent Bruce Ctrl-cKO littermate pairs. Bars, $5 \mu \mathrm{m}$. c Quantification of MLH1 foci in Bruce Ctrl and cKO mid-late pachytene spermatocytes. There is no statistical difference of MLH1 foci between Bruce Ctrl and cKO spermatocytes. Data are aggregated from three independent Bruce Ctrl-cKO littermate pairs. Horizontal line, mean. Student's $t$ test, n.s. not significant, $p>0.05$. d Chromosomal fragmentation in Bruce cKO spermatocytes immunostained with SYCP3 (green) and $\gamma \mathrm{H} 2 \mathrm{AX}$ (red) antibodies. A representative Bruce cKO mid pachytene spermatocyte is shown to have 20 pairs of chromosomes plus one additional chromosome fragment associated with strong $\gamma \mathrm{H} 2 \mathrm{AX}$ immunofluorescence. The chromosomal fragment in the dashed rectangle is magnified to the right. Representative image from two independent

Ctrl (Fig. 6h). However, the pachytene signals were largely devoid from both the autosome fragments (Fig. 6i) and the radials (Fig. 6j, k), suggesting a MSUC defect.

To confirm this ATR signaling defect, we examined two HORMA domain proteins, HORMAD1 and HORMAD2 that localize on unsynapsed chromosomes to regulate meiotic silencing by promoting efficient ATR activity [46, 49-52]. In comparison to HORMAD1 recruitment patterns in Ctrl zygotene (Fig. 7a) and pachytene (Fig. 7b) spermatocytes, there was no distinguishable change in recruitment patterns in cKO zygotene cells (Fig. 7c), but reduced accumulation of HORMAD1 to the unsynapsed regions of quadrivalents in Bruce cKO pachytene cells (Fig. 7d). Similar results were observed for HORMAD2 in
Bruce Ctrl-cKO littermate pairs. Bar, $5 \mu \mathrm{m}$. (e, f) Chromosomal axial damage in Bruce cKO pachytene spermatocytes immunostained with SYCP3 (green) and $\gamma \mathrm{H} 2 \mathrm{AX}$ (red) antibodies. e A representative Bruce cKO mid pachytene spermatocyte is shown to have a chromosomal axial structure that is discontinuous and without $\gamma \mathrm{H} 2 \mathrm{AX}$ around the breaking point (white arrow). f A representative Bruce cKO mid pachytene spermatocyte is shown to bear a discontinuous axis as above, except with prominent $\gamma \mathrm{H} 2 \mathrm{AX}$ signals around the break point (white arrow). Chromosomal axial damage in the dashed rectangles are magnified to the right. Representative images from two independent Bruce Ctrl-cKO littermate pairs. Bars, $5 \mu \mathrm{m}$. g Frequency of chromosomal fragments and axial damage in Bruce Ctrl and cKO pachytene spermatocytes. Data are aggregated from two independent Bruce Ctrl-cKO littermate pairs (Ctrl, $n=441$; cKO, $n=575$ ). Error bar, standard deviation. Student's $t$ test, $* * * p<0.001$.

Ctrl (Fig. 7e, f) and cKO (Fig. 7g-i). These results support the notion of impaired ATR signaling in MSUC at quadrivalents. Supporting the requirement of Bruce for MSUC, a phosphorylated form of RNA polymerase II (POL II), a marker for active transcription [53], was abnormally present on the cKO unsynapsed segments of quadrivalents (Fig. 7k), indicating that such sites are not silenced. On the other hand, the absence of POL II staining from the XY body (Fig. 7j, k) showed a prominent MSCI in both Bruce Ctrl and cKO.

To verify the integrity of MSCI in cKO pachytene spermatocytes, we performed gene-specific RNA FISH to detect the transcription of Lamp2, an X-linked gene expressed in spermatogonia, whereas silenced in pachytene 

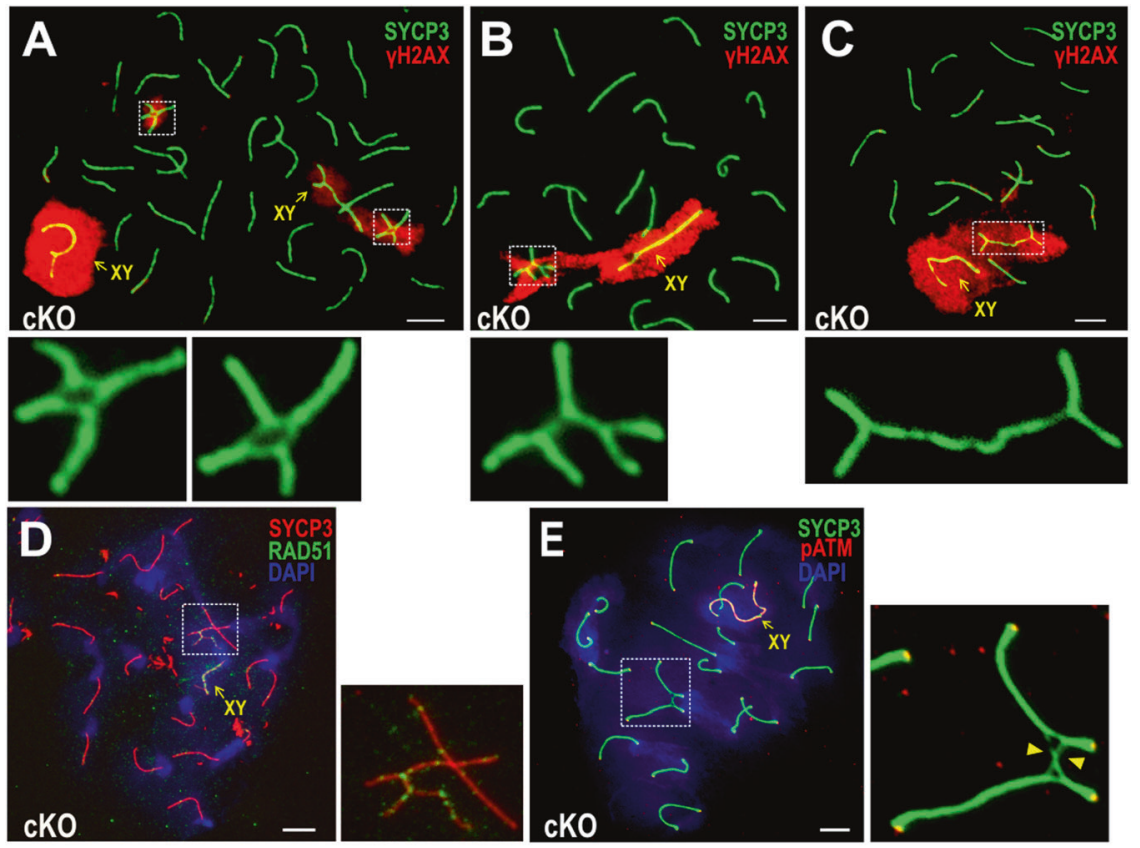

\begin{tabular}{lr} 
F & Bruce Ctr \\
\hline Translocation & $0.16 \%$ \\
Asynapsis & $1.87 \%$ \\
Autosomal $\mathrm{yH} 2 \mathrm{AX}$ & $5.25 \%$ \\
Fragment \& axial damage & $0.19 \%$ \\
\hline
\end{tabular}

Fig. 5 Chromosomal radials and translocations in Bruce cKO spermatocytes. a-c Chromosome spreads of Bruce cKO pachytene spermatocytes immunostained with SYCP3 (green) and $\gamma \mathrm{H} 2 \mathrm{AX}$ (red) antibodies. Two nonhomologous chromosomes form radial structures; these structures are notable for colocalization of intense $\gamma \mathrm{H} 2 \mathrm{AX}$ signals and are frequently seen near to or adjacent to the XY body, the chromo-nuclear compartment enveloping the $\mathrm{X}$ and $\mathrm{Y}$ chromosomes in the pachytene and diplotene stages of meiotic prophase I. Based on radial conformation, three types of chromosomal translocation could be distinguished: reciprocal (a), nonreciprocal (b), and Robertsonianlike (c). Chromosomal radials outlined in the dashed rectangles are magnified in the bottom panels. Representative images from four independent Bruce Ctrl-cKO littermate pairs. Bars, $5 \mu \mathrm{m}$. d Chromosomal spread of a Bruce cKO pachytene spermatocyte immunostained with SYCP3 (red) and RAD51 (green) antibodies. Chromosomal radials in Bruce cKO spermatocytes are RAD51 (green) intensive, implying uncompleted meiotic homologous recombination. The

spermatocytes as a result of MSCI [54]. In contrast to the expected Lamp2 signals in spermatogonia, no such signals were observed in Bruce Ctrl and cKO pachytene spermatocytes (Fig. S4D). Further, we performed RNA FISH for Cot-1. Cot-1 DNA consists of repetitive elements that will hybridize efficiently to nascent transcripts, enabling visualization of transcriptionally active regions and thus can evaluate transcription status in MSCI [21]. In both samples, Cot-1 RNA FISH signals are excluded from DAPI-intense heterochromatin and the XY body (Fig. S4E), suggesting that MSCI is maintained even without Bruce.

\section{SC structural defects are associated with multivalent chromosome radials}

In pachytene meiocytes, SC structures are critical for the maintenance of homolog interactions. To investigate the effect of Bruce deficiency on SC structures, we analyzed the SCs of quadrivalents and hexavalents in Bruce cKO mice via dual immunofluorescence for the SC axial element protein SYCP3 and the SC central element protein SYCP1. SYCP1 is present only at synapsed axes, while SYCP3 is present at both synapsed and unsynapsed axes. In Bruce 


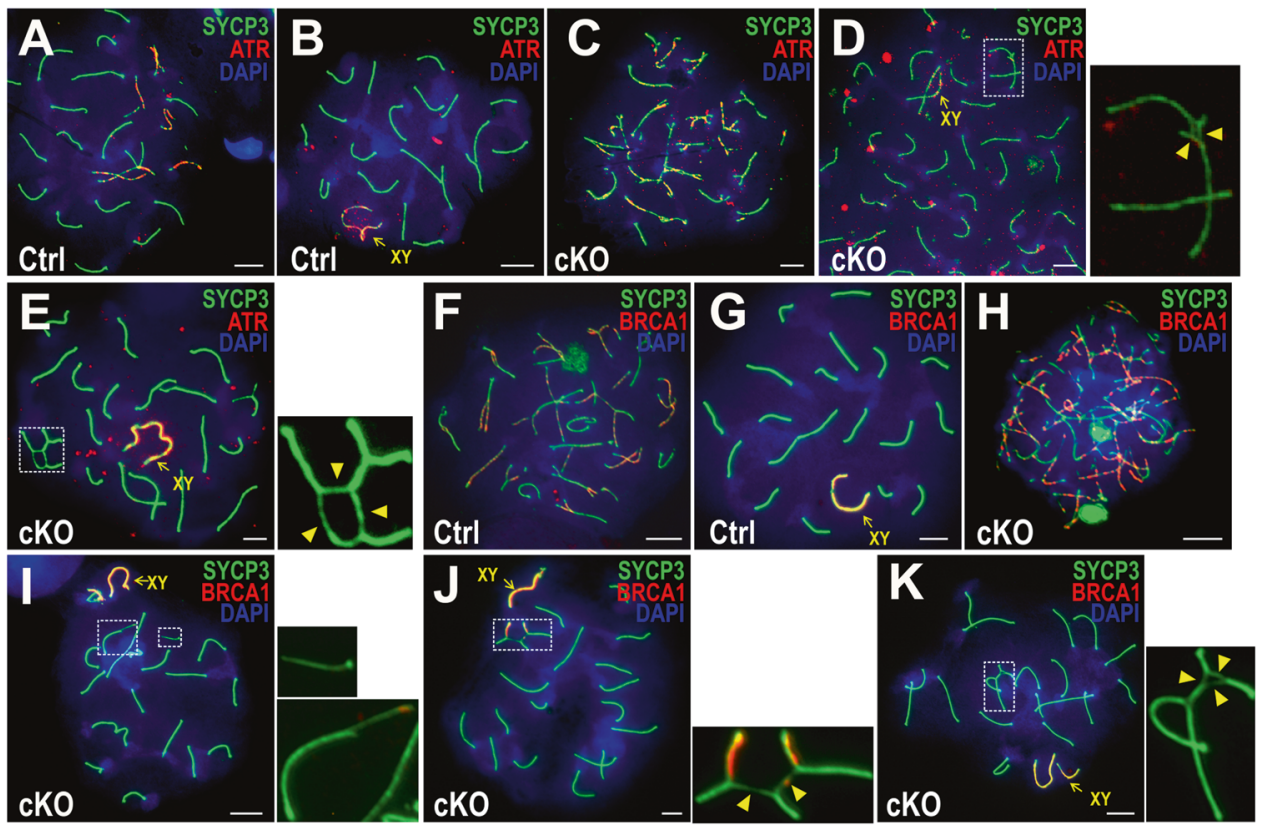

Fig. 6 Impaired ATR and BRCA1 signals in Bruce cKO spermatocytes with chromosomal translocations. Immunofluorescence (IF) staining of SYCP3 (green) and ATR (red) in chromosome spreads of Bruce Ctrl and cKO spermatocytes in the zygotene (a, c) and pachytene (b, d, e) stages. We observed the normal formation of ATR foci in Bruce cKO zygotene spermatocytes (c), the partial loss of ATR signals at unsynapsed chromatin within the radials of Bruce cKO mid pachytene spermatocytes (d), and total loss of ATR signals at unsynapsed segments in Bruce cKO late pachytene spermatocytes (e). The chromosomal radials within the dashed rectangles are magnified to the right (arrowheads, unsynapsed chromosomal segments). Note that ATR signals on the sex chromosomes (yellow arrows) are also reduced (also see Fig. S4A-C). Representative images from two independent Bruce Ctrl-cKO littermate pairs (cKO spermatocytes bearing radials, $n=21$ ). Bars, $5 \mu \mathrm{m}$. IF staining of SYCP3 (green) and a marker of chromosomal synapsis, BRCA1 (red), in chromosome spreads of Bruce Ctrl and cKO spermatocytes in the zygotene $(\mathbf{f}, \mathbf{h})$ and mid

cKO, the unsynapsed regions of quadrivalents (Fig. 8a, b) and hexavalents (Fig. 8c) bore SYCP3 signals but lacked SYCP1 signals. Intriguingly, hexavalents usually involve one pair of sex chromosomes and two pairs of autosomes (Fig. 8c). To confirm the absence of SYCP1 signals on rearranged chromosomes, we have presented a representative image with multiple nuclei bearing translocations (Fig. 8d). The weaker or absent SYCP1 signals indicate structural defects in the SC of rearranged chromosomes.

\section{Discussion}

In mitotic cells, BRUCE facilitates the ATM-DNA-DSBmediated HR repair of DNA DSBs for genomic stability. This function is mediated in a BRUCE E2/E3 ligase-dependent manner that facilitates the localization of BRIT1/MCPH1 and the chromatin remodeler SWI-SNF to DSBs $[8,9]$. BRUCE also facilitates activation of the ATR-DNA replication stress pachytene (g, i) stages. BRCA1 accumulation patterns appear normal in Bruce cKO zygotene spermatocyte (h), while are largely lost in Bruce cKO pachytene spermatocytes bearing chromosomal fragments (i), which are bounded by dashed rectangles and magnified to the right. Note that BRCA1 signals are largely or entirely absent from the $\mathrm{cKO}$ mid pachytene chromosomal fragments. Representative images from two independent Bruce Ctrl-cKO littermate pairs. Bars, $5 \mu \mathrm{m}$. (j, k) IF staining of SYCP3 (green) and BRCA1 (red) in Bruce cKO spermatocytes bearing chromosomal translocations in the mid pachytene (j) and late pachytene (k) stages. Chromosomal radials in the dashed rectangles are magnified to the right. Note that, within the radials, BRCA1 signals are partially lost from unsynapsed regions in the mid pachytene stage and entirely lost from such regions in the late pachytene stage (arrowheads), while BRCA1 signal appears normal on the sex chromosomes in both stages (yellow arrows). Representative images from three independent Bruce Ctrl-cKO littermate pairs (cKO spermatocytes bearing radials, $n=16$ ). Bars, $5 \mu \mathrm{m}$.

response to protect against replication fork collapse and the activation of new replication origins [14]. The current study provides the first evidence for a critical function for BRUCE in the maintenance of genomic stability in germ cells, particularly in spermatogonia and spermatocytes. We have characterized spermatogenesis in Bruce cKO mice and demonstrated that Bruce is required for (i) the maintenance of spermatogonia and (ii) the protection of genomic stability by promoting ATM and ATR DDR for the repair of physiologically induced DNA DSBs and homolog synapsis. The loss of Bruce in the testes reduces the number of spermatogonia and induces genomic instability in the form of chromosomal translocation, fragmentation, axial damage and aneuploidy. Consequently, massive spermatocyte loss through cell death gives rise to smaller testis sizes and largely empty epididymides in Bruce cKO.

These findings, which center on the function of Bruce in spermatogonia maintenance and spermatogenesis, are important for understanding the maintenance of genomic stability in 

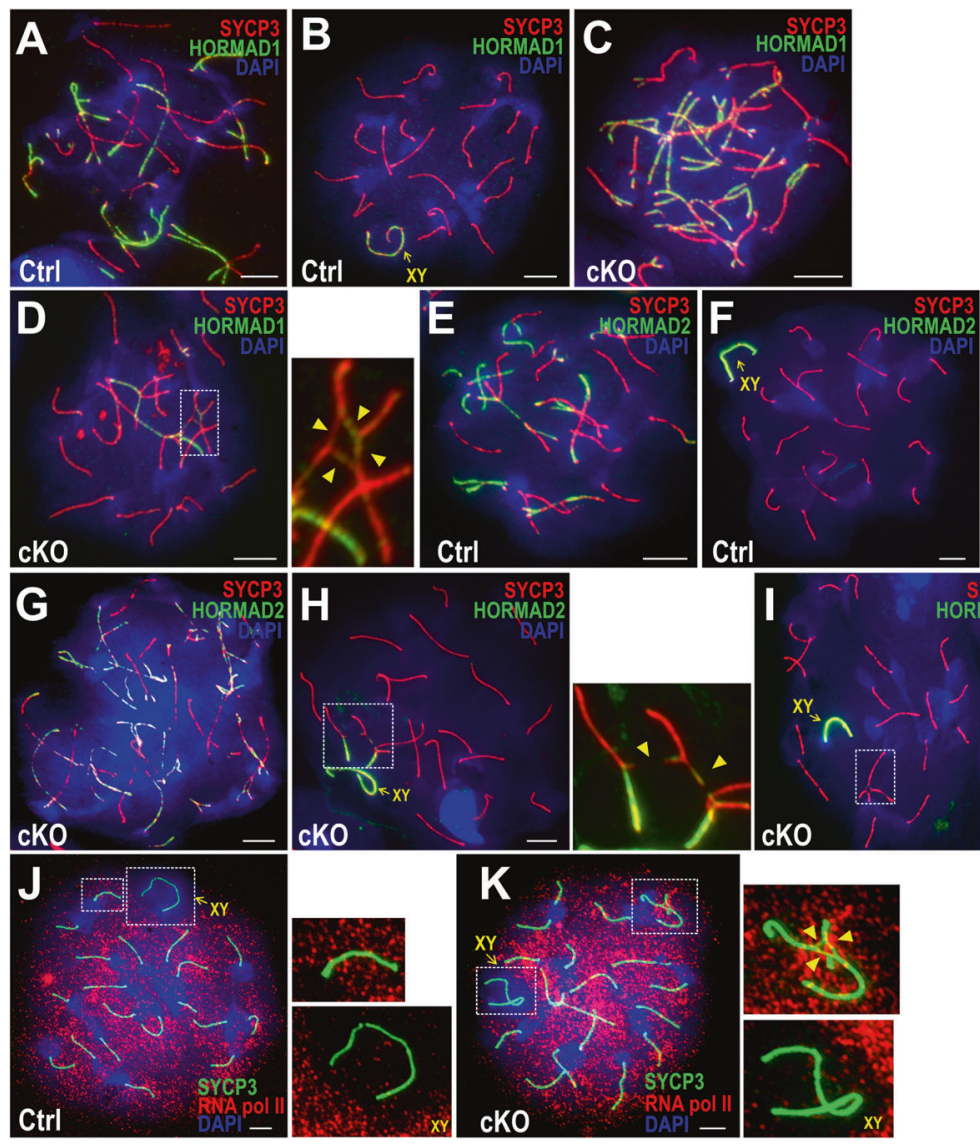

Fig. 7 HORMAD1 and HORMAD2 are lost in chromosomal radials in Bruce cKO. IF staining of SYCP3 (red) and HORMAD1 (green) in Bruce Ctrl spermatocytes in the zygotene (a) and pachytene (b) stages. Representative images from two independent Bruce Ctrl mice. Bars, $5 \mu \mathrm{m}$. IF staining of SYCP3 (red) and HORMAD1 (green) in Bruce cKO spermatocytes in the zygotene (c) and pachytene (d) stages. Chromosomal radials in the dashed rectangles are magnified to the right with unsynapsed chromosomal segments indicated by arrowheads. Representative images from two independent Bruce cKO mice (cKO spermatocytes bearing radials, $n=11$ ). Bars, $5 \mu \mathrm{m}$. IF staining of SYCP3 (red) and HORMAD2 (green) in Bruce Ctrl spermatocytes in the zygotene (e) and pachytene (f) stages. Representative images from two independent Bruce Ctrl mice. Bars, $5 \mu \mathrm{m}$. IF staining of SYCP3 (red) and HORMAD2 (green) in Bruce cKO spermatocytes in the zygotene ( $\mathbf{g})$ and pachytene $(\mathbf{h}, \mathbf{i})$ stages. Chromosomal radials in the dashed rectangles are magnified to the right; arrowheads point to unsynapsed chromosomal segments in open (h) and closed radials (i).

germ cells. The quadrivalents and hexavalents in Bruce cKO testes closely resemble Robertsonian translocations, which are the most common forms of autosome translocation in humans causing male reproductive defects [37, 38].

Far less is known about how genomic stability is maintained during meiosis than in somatic cells. Given that deregulation of genomic integrity in meiosis can contribute to sterility or reduced fertility in males, the regulation of BRUCE activity in controlling genomic stability provides the rationale for BRUCE being a candidate gene that is mutated or inactivated in certain human reproductive disorders. To this
Representative images from two independent Bruce cKO mice (cKO spermatocytes bearing radials, $n=9$ ). Bars, $5 \mu \mathrm{m}$. (j, k) IF staining of SYCP3 (green) and anti-RNA polymerase II (RNA Pol II; red) antibodies in Bruce Ctrl and cKO pachytene spermatocytes carrying chromosomal translocations. The sex chromosomes in the Ctrl form a silent compartment, the XY body, in which RNA Pol II signals are largely excluded ( $\mathbf{j}$, lower right); meanwhile, fully synapsed autosomes are positive for RNA Pol II signals (j, upper right). In Bruce cKO, the nuclear compartment containing chromosomal radials (k, upper right) bears strong RNA Pol II signals and thus an apparent failure to catalyze MSUC. The sex chromosomes in cKO resides in a silent XY body, which is devoid of RNA Pol II foci, thereby indicating the presence of MSCI ( $\mathbf{k}$, lower right). The arrowheads in $\mathbf{k}$ indicate unsynapsed regions. Representative images from two independent Bruce Ctrl-cKO littermate pairs (cKO spermatocytes bearing radials, $n=15)$. Bars, $5 \mu \mathrm{m}$.

end, our Bruce cKO mouse model offers an attractive experimental tool to address the underlying pathogenic processes of this long-standing question. In somatic cells, chromosomal fragmentation and translocation (either genetically acquired or acquired later in life) are hallmarks of cancer, and are exemplified in Ataxia telangiectasia, Nijmegen breakage syndrome, Bloom syndrome, and Werner syndrome [55, 56]. However, the cause of chromosomal translocation remains elusive. DNA breaks are considered a prerequisite for chromosome fragmentation and translocation [57, 58]. Since Bruce-deficient germ and somatic cells have aberrant 

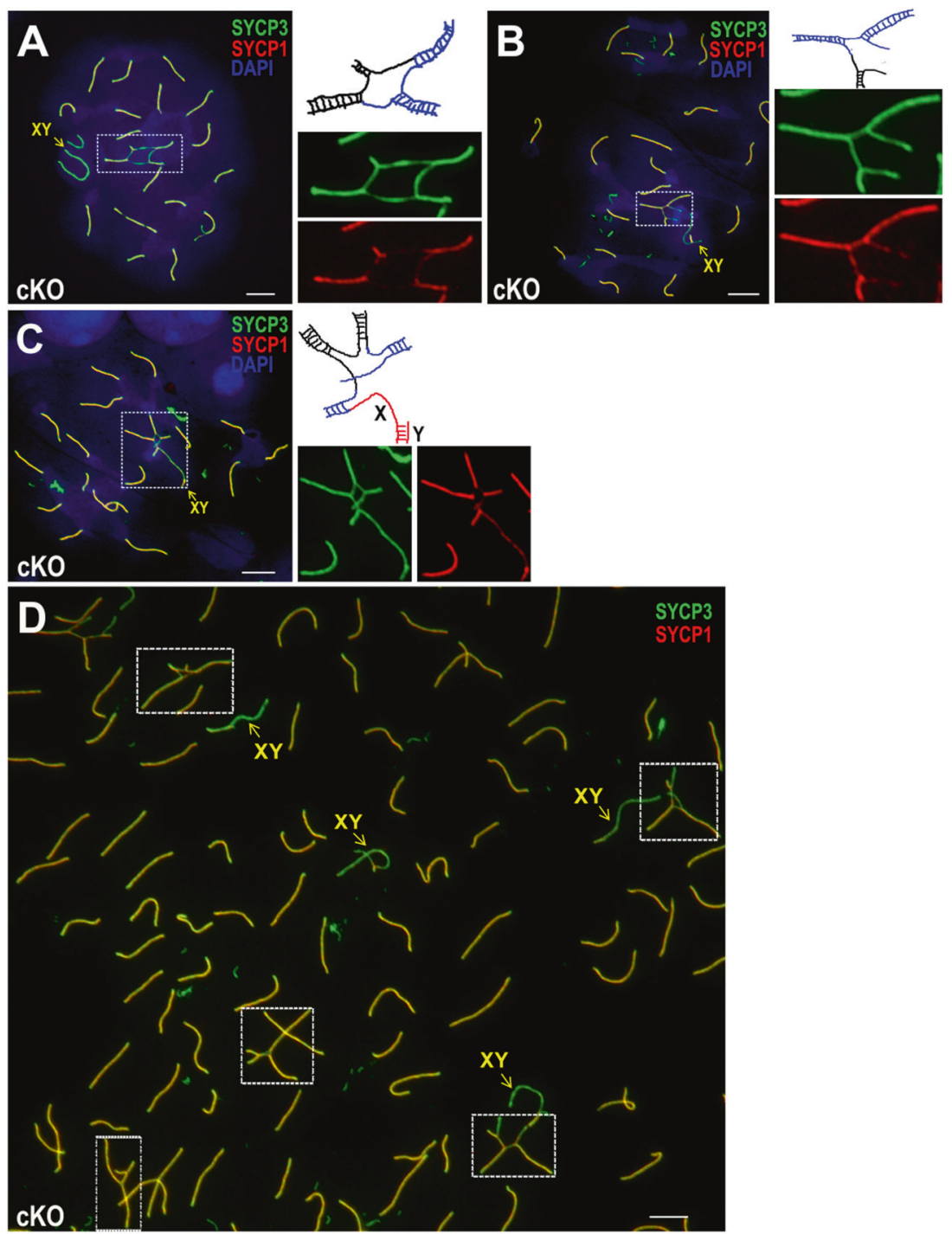

Fig. 8 Full synapsis is disturbed by chromosomal translocation in Bruce cKO. (a-c) Chromosome spreads of Bruce cKO spermatocytes immunostained with SYCP3 (green), a marker of the synaptonemal complex (SC) axial element, and SYCP1 (red), a marker of the SC central element. Representative image of a cKO late pachytene spermatocyte bearing a quadrivalent translocation formed by reciprocal translocation (a). Representative image of a cKO early pachytene spermatocyte bearing a quadrivalent translocation formed via nonreciprocal translocation (b). Representative image of a cKO early pachytene spermatocyte bearing a hexavalent translocation involving autosomes and sex chromosomes (c). The chromosomal radials outlined in the dashed rectangles are magnified in the right panels.

accumulation of DSBs and structural damage, further studies of Bruce function could provide an opportunity for understanding Bruce-regulated signaling in protection against chromosome fragility.

In addition to meiotic DNA break repair and homolog synapsis, Bruce may also influence MSUC [44, 59]. The persistence of RNA Pol II on unsynapsed chromosome segments in Bruce cKO spermatocytes is consistent with a defect in MSUC. However, the initiation of MSCI appears
Illustrations of the translocations are provided as a simple interpretation of configurations, although other interpretations are also possible. Railway-like structures indicate the SC axial and central elements. Representative images from three independent Bruce cKO mice (cKO spermatocytes bearing radials, $n=33$ ). Bars, $5 \mu \mathrm{m}$. d In a large visual field of a Bruce cKO chromosome spread of early and mid pachytene spermatocytes, multiple chromosomal radials with unsynapsed segments are observable. This image is further indication of the relatively high frequency of chromosomal translocations in spermatocytes deficient for BRUCE. The chromosomal radials are outlined in the dashed rectangles. Representative image from three independent Bruce cKO mice. Bar, $5 \mu \mathrm{m}$.

not to be affected in Bruce cKO spermatocytes, suggesting that Bruce may be involved in the maintenance of MSUC through the ATR-DDR pathway. In support of this notion, ATR signal intensity on the XY body was decreased in Bruce cKO spermatocytes after the mid pachytene stage compared with the Ctrl. Usually, meiotic cells with severe DNA damage, asynapsis, and impaired MSUC trigger meiotic checkpoints. However, such cells in Bruce cKO testes may be subject to an impaired 
checkpoint since they still progress through pachytene and enter the diplotene stage (Fig. 3i). Therefore, Bruce could also be crucial for maintaining meiotic checkpoints.

Since Bruce cKO testes displayed both increased apoptosis and meiotic defects, we were presented with an intriguing question: Are the depleted germ cells resulted primarily from the loss of Bruce antiapoptotic activity or from meiotic arrest? Bruce function may be related to other DDR factors including Blm, Slx4, Fancd2, and Fkbp6, since these mutants exhibit similar rearranged autosome structures in their respective $\mathrm{KO}$ mice [60-67]. We favor a working model in which loss of germ cells in Bruce mutant mice can be attributed to pachytene spermatocyte apoptosis induced by meiotic defects, and that apoptosis is secondary to DSBs and chromosomal instability. Deficiency of Bruce perhaps makes the germ cells prone to apoptosis. Upon DNA damage, the germ cells will undergo apoptosis and be eliminated. While uncoupling the two activities might be challenging, new approaches could become available in the future.

Acknowledgements We thank Dr Attila Tóth for providing the HORMAD1 and HORMAD2 antibodies, and Dr James Ingres for providing the RPA1 antibody. This work was supported by NIH grants CA158323, CA158323S, and CA241025 (CD); GM098605 (SHN).

\section{Compliance with ethical standards}

Conflict of interest The authors declare that they have no conflict of interest.

Publisher's note Springer Nature remains neutral with regard to jurisdictional claims in published maps and institutional affiliations.

\section{References}

1. Chen Z, Naito M, Hori S, Mashima T, Yamori T, Tsuruo T. A human IAP-family gene, apollon, expressed in human brain cancer cells. Biochem Biophys Res Commun. 1999;264:847-54.

2. Lotz K, Pyrowolakis G, Jentsch S. BRUCE, a Giant E2/E3 Ubiquitin Ligase and Inhibitor of Apoptosis Protein of the trans-Golgi Network, Is Required for Normal Placenta Development and Mouse Survival. Mol Cell Biol. 2004;24:9339-50.

3. Bartke T, Pohl C, Pyrowolakis G, Jentsch S. Dual role of BRUCE as an antiapoptotic IAP and a chimeric E2/E3 ubiquitin ligase. Mol Cell. 2004;14:801-11.

4. Hauser HP, Bardroff M, Pyrowolakis G, Jentsch S. A giant ubiquitin-conjugating enzyme related to IAP apoptosis inhibitors. J Cell Biol. 1998;141:1415-22.

5. Hao Y, Sekine K, Kawabata A, Nakamura H, Ishioka T, Ohata H, et al. Apollon ubiquitinates SMAC and caspase-9, and has an essential cytoprotection function. Nat Cell Biol. 2004;6:849-60.

6. Ren J, Shi M, Liu R, Yang QH, Johnson T, Skarnes WC, et al. The Birc6 (Bruce) gene regulates p53 and the mitochondrial pathway of apoptosis and is essential for mouse embryonic development. Proc Natl Acad Sci U S A. 2005;102:565-70.

7. Pohl C, Jentsch S. Final stages of cytokinesis and midbody ring formation are controlled by BRUCE. Cell. 2008;132:832-45.

8. Ge C, Che L, Du C. The UBC Domain Is Required for BRUCE to Promote BRIT1/MCPH1 Function in DSB Signaling and Repair
Post Formation of BRUCE-USP8-BRIT1 Complex. PLoS One. 2015;10:e0144957.

9. Ge C, Che L, Ren J, Pandita RK, Lu J, Li K, et al. BRUCE regulates DNA double-strand break response by promoting USP8 deubiquitination of BRIT1. Proc Natl Acad Sci USA. 2015;112: E1210-9.

10. Bakkenist CJ, Kastan MB. Initiating cellular stress responses. Cell. 2004;118:9-17.

11. Marechal A, Zou L. DNA damage sensing by the ATM and ATR kinases. Cold Spring Harb Perspect Biol. 2013;5:pii: a012716. https://doi.org/10.1101/cshperspect.a012716.

12. Kastan MB, Bartek J. Cell-cycle checkpoints and cancer. Nature. 2004;432:316-23.

13. Ciccia A, Elledge SJ. The DNA damage response: making it safe to play with knives. Mol Cell. 2010;40:179-204.

14. Ge C, Vilfranc CL, Che L, Pandita RK, Hambarde S, Andreassen $\mathrm{PR}$, et al. The BRUCE-ATR signaling axis is required for accurate DNA replication and suppression of liver cancer development. Hepatology. 2019;69:2608-22.

15. Phillips BT, Gassei K, Orwig KE. Spermatogonial stem cell regulation and spermatogenesis. Philos Trans R Soc Lond B Biol Sci. 2010;365:1663-78.

16. Handel MA, Schimenti JC. Genetics of mammalian meiosis: regulation, dynamics and impact on fertility. Nat Rev Genet. 2010;11:124-36.

17. Hunter N. Meiotic Recombination: The Essence of Heredity. Cold Spring Harb Perspect Biol. 2015;7:a016618.

18. Alavattam KG, Abe H, Sakashita A, Namekawa SH. Chromosome Spread Analyses of Meiotic Sex Chromosome Inactivation. Methods Mol Biol. 2018;1861:113-29.

19. Alavattam KG, Kato Y, Sin HS, Maezawa S, Kowalski IJ, Zhang F, et al. Elucidation of the Fanconi Anemia Protein Network in Meiosis and Its Function in the Regulation of Histone Modifications. Cell reports. 2016;17:1141-57.

20. Namekawa SH Slide preparation method to preserve threedimensional chromatin architecture of testicular germ cells. Journal of visualized experiments: JoVE. 2014; e50819.

21. Namekawa SH, Park PJ, Zhang LF, Shima JE, McCarrey JR, Griswold MD, et al. Postmeiotic sex chromatin in the male germline of mice. Curr Biol. 2006;16:660-7.

22. Namekawa SH, Lee JT. Detection of nascent RNA, single-copy DNA and protein localization by immunoFISH in mouse germ cells and preimplantation embryos. Nat Protoc. 2011;6:270-84.

23. Namekawa SH, Payer B, Huynh KD, Jaenisch R, Lee JT. Twostep imprinted $\mathrm{X}$ inactivation: repeat versus genic silencing in the mouse. Mol Cell Biol. 2010;30:3187-205.

24. Ichijima Y, Ichijima M, Lou Z, Nussenzweig A, Camerini-Otero $\mathrm{RD}$, Chen J, et al. MDC1 directs chromosome-wide silencing of the sex chromosomes in male germ cells. Genes Dev. 2011;25: 959-71.

25. Ruzankina Y, Pinzon-Guzman C, Asare A, Ong T, Pontano L, Cotsarelis G, et al. Deletion of the developmentally essential gene ATR in adult mice leads to age-related phenotypes and stem cell loss. Cell Stem Cell. 2007;1:113-26.

26. Chaboissier MC, Kobayashi A, Vidal VI, Lutzkendorf S, van de Kant HJ, Wegner M, et al. Functional analysis of Sox8 and Sox9 during sex determination in the mouse. Development. 2004;131:1891-901.

27. Chang H, Gao F, Guillou F, Taketo MM, Huff V, Behringer RR. Wt1 negatively regulates beta-catenin signaling during testis development. Development. 2008;135:1875-85.

28. Costoya JA, Hobbs RM, Barna M, Cattoretti G, Manova K, Sukhwani M, et al. Essential role of Plzf in maintenance of spermatogonial stem cells. Nat Genet. 2004;36:653-9.

29. Buaas FW, Kirsh AL, Sharma M, McLean DJ, Morris JL, Griswold MD, et al. Plzf is required in adult male germ cells for stem cell self-renewal. Nat Genet. 2004;36:647-52. 
30. Takase HM, Nusse R. Paracrine Wnt/beta-catenin signaling mediates proliferation of undifferentiated spermatogonia in the adult mouse testis. Proc Natl Acad Sci U S A. 2016;113:E1489-97.

31. Schrans-Stassen BH, van de Kant HJ, de Rooij DG, van Pelt AM. Differential expression of c-kit in mouse undifferentiated and differentiating type A spermatogonia. Endocrinology. 1999; 140:5894-900.

32. Mahadevaiah SK, Turner JM, Baudat F, Rogakou EP, de Boer P, Blanco-Rodriguez J, et al. Recombinational DNA double-strand breaks in mice precede synapsis. Nat Genet. 2001;27:271-6.

33. Lange J, Pan J, Cole F, Thelen MP, Jasin M, Keeney S. ATM controls meiotic double-strand-break formation. Nature. 2011;479: 237-40.

34. Bannister LA, Schimenti JC. Homologous recombinational repair proteins in mouse meiosis. Cytogenet Genome Res. 2004;107: 191-200.

35. Wold MS. Replication protein A: a heterotrimeric, single-stranded DNA-binding protein required for eukaryotic DNA metabolism. Annual review of biochemistry. 1997;66:61-92.

36. Baker SM, Plug AW, Prolla TA, Bronner CE, Harris AC, Yao X, et al. Involvement of mouse Mlh1 in DNA mismatch repair and meiotic crossing over. Nat Genet. 1996;13:336-42.

37. Oliver-Bonet M, Benet J, Sun F, Navarro J, Abad C, Liehr T, et al. Meiotic studies in two human reciprocal translocations and their association with spermatogenic failure. Hum Reprod. 2005; 20:683-8.

38. Berrios S, Manieu C, Lopez-Fenner J, Ayarza E, Page J, Gonzalez $\mathrm{M}$, et al. Robertsonian chromosomes and the nuclear architecture of mouse meiotic prophase spermatocytes. Biol Res. 2014;47:16.

39. Jiang H, Wang L, Cui Y, Xu Z, Guo T, Cheng D, et al. Meiotic chromosome behavior in a human male $t(8 ; 15)$ carrier. J Genet Genomics. 2014;41:177-85.

40. Roukos V, Misteli T. The biogenesis of chromosome translocations. Nat Cell Biol. 2014;16:293-300.

41. Meaburn KJ, Misteli T, Soutoglou E. Spatial genome organization in the formation of chromosomal translocations. Semin Cancer Biol. 2007; 17:80-90.

42. Pacheco S, Maldonado-Linares A, Marcet-Ortega M, Rojas C, Martinez-Marchal A, Fuentes-Lazaro J, et al. ATR is required to complete meiotic recombination in mice. Nature communications. 2018;9:2622.

43. Widger A, Mahadevaiah SK, Lange J, ElInati E, Zohren J, Hirota $\mathrm{T}$, et al. ATR is a multifunctional regulator of male mouse meiosis. Nature communications. 2018;9:2621.

44. Turner JM. Meiotic Silencing in Mammals. Annual review of genetics. 2015;49:395-412.

45. Ichijima Y, Sin HS, Namekawa SH. Sex chromosome inactivation in germ cells: emerging roles of DNA damage response pathways. Cell Mol Life Sci. 2012;69:2559-72.

46. Royo H, Prosser H, Ruzankina Y, Mahadevaiah SK, Cloutier JM, Baumann M, et al. ATR acts stage specifically to regulate multiple aspects of mammalian meiotic silencing. Genes Dev. 2013;27: 1484-94.

47. Turner JM, Aprelikova O, Xu X, Wang R, Kim S, Chandramouli $\mathrm{GV}$, et al. BRCA1, histone $\mathrm{H} 2 \mathrm{AX}$ phosphorylation, and male meiotic sex chromosome inactivation. Curr Biol. 2004;14:2135-42.

48. Broering TJ, Alavattam KG, Sadreyev RI, Ichijima Y, Kato Y, Hasegawa K, et al. BRCA1 establishes DNA damage signaling and pericentric heterochromatin of the $\mathrm{X}$ chromosome in male meiosis. J Cell Biol. 2014;205:663-75.

49. Daniel K, Lange J, Hached K, Fu J, Anastassiadis K, Roig I, et al. Meiotic homologue alignment and its quality surveillance are controlled by mouse HORMAD1. Nat Cell Biol. 2011;13:599-610.

50. Kogo H, Tsutsumi M, Inagaki H, Ohye T, Kiyonari H, Kurahashi H. HORMAD2 is essential for synapsis surveillance during meiotic prophase via the recruitment of ATR activity. Genes Cells. 2012;17:897-912.

51. Shin YH, Choi Y, Erdin SU, Yatsenko SA, Kloc M, Yang F, et al. Hormad1 mutation disrupts synaptonemal complex formation, recombination, and chromosome segregation in mammalian meiosis. PLoS Genet. 2010;6:e1001190.

52. Wojtasz L, Daniel K, Roig I, Bolcun-Filas E, Xu H, Boonsanay V, et al. Mouse HORMAD1 and HORMAD2, two conserved meiotic chromosomal proteins, are depleted from synapsed chromosome axes with the help of TRIP13 AAA-ATPase. PLoS Genet. 2009;5: e1000702.

53. Page J, de la Fuente R, Manterola M, Parra MT, Viera A, Berrios $\mathrm{S}$, et al. Inactivation or non-reactivation: what accounts better for the silence of sex chromosomes during mammalian male meiosis? Chromosoma. 2012;121:307-26.

54. Hasegawa K, Sin HS, Maezawa S, Broering TJ, Kartashov AV, Alavattam KG, et al. SCML2 establishes the male germline epigenome through regulation of histone $\mathrm{H} 2 \mathrm{~A}$ ubiquitination. Dev Cell. 2015;32:574-88.

55. Duker NJ. Chromosome breakage syndromes and cancer. Am J Med Genet. 2002;115:125-9.

56. Nambiar M, Kari V, Raghavan SC. Chromosomal translocations in cancer. Biochim Biophys Acta. 2008;1786:139-52.

57. Lobachev K, Vitriol E, Stemple J, Resnick MA, Bloom K. Chromosome fragmentation after induction of a double-strand break is an active process prevented by the RMX repair complex. Curr Biol. 2004;14:2107-12.

58. Gostissa M, Alt FW, Chiarle R. Mechanisms that promote and suppress chromosomal translocations in lymphocytes. Annu Rev Immunol. 2011;29:319-50.

59. Turner JM, Mahadevaiah SK, Fernandez-Capetillo O, Nussenzweig A, Xu X, Deng CX, et al. Silencing of unsynapsed meiotic chromosomes in the mouse. Nat Genet. 2005;37:41-7.

60. Liang Y, Gao H, Lin SY, Peng G, Huang X, Zhang P, et al. BRIT1/MCPH1 is essential for mitotic and meiotic recombination DNA repair and maintaining genomic stability in mice. PLoS Genet. 2010;6:e1000826.

61. Vasileva A, Hopkins KM, Wang X, Weisbach MM, Friedman RA, Wolgemuth DJ, et al. The DNA damage checkpoint protein RAD9A is essential for male meiosis in the mouse. J Cell Sci. 2013;126:3927-38.

62. Yoshida K, Kondoh G, Matsuda Y, Habu T, Nishimune Y, Morita T. The mouse RecA-like gene Dmc1 is required for homologous chromosome synapsis during meiosis. Mol Cell. 1998;1:707-18.

63. Holloway JK, Morelli MA, Borst PL, Cohen PE. Mammalian BLM helicase is critical for integrating multiple pathways of meiotic recombination. J Cell Biol. 2010;188:779-89.

64. Crackower MA, Kolas NK, Noguchi J, Sarao R, Kikuchi K, Kaneko H, et al. Essential role of Fkbp6 in male fertility and homologous chromosome pairing in meiosis. Science. 2003; 300:1291-5.

65. Houghtaling S, Timmers C, Noll M, Finegold MJ, Jones SN, Meyn MS, et al. Epithelial cancer in Fanconi anemia complementation group D2 (Fancd2) knockout mice. Genes Dev. 2003;17:2021-35.

66. Wong JC, Alon N, McKerlie C, Huang JR, Meyn MS, Buchwald M. Targeted disruption of exons 1 to 6 of the Fanconi Anemia group A gene leads to growth retardation, strain-specific microphthalmia, meiotic defects and primordial germ cell hypoplasia. Hum Mol Genet. 2003;12:2063-76.

67. Holloway JK, Mohan S, Balmus G, Sun X, Modzelewski A, Borst PL, et al. Mammalian BTBD12 (SLX4) protects against genomic instability during mammalian spermatogenesis. PLoS Genet. 2011;7:e1002094. 